\title{
ZONEAMENTO AGROCLIMÁTICO PARA ESPÉCIES DO GÊNERO Pinus NO ESTADO DO ESPÍRITO SANTO
}

\author{
Fábio da Silveira Castro ${ }^{1}$, José Eduardo Macedo Pezzopane ${ }^{2}$, José Ricardo Macedo Pezzopane ${ }^{3}$, \\ Roberto Avelino Cecílio ${ }^{4}$, Alexandre Cândido Xavier ${ }^{5}$ \\ ${ }^{1}$ Eng. Agrônomo, M.Sc., Alegre, ES, Brasil - fabiosilveira_70@hotmail.com \\ ${ }^{2}$ Eng. Florestal, Dr., Depto. de Engenharia Rural, UFES, Alegre, ES, Brasil - pezzopane@ reitoria.ufes.br \\ ${ }^{3}$ Eng. Agrônomo, Dr., Depto. Ciências da Saúde, Biológicas e Agrárias, UFES, Alegre, ES, Brasil - josepezzopane@ @eunes.ufes.br \\ ${ }^{4}$ Eng. Agrícola, Dr., Depto. de Engenharia Florestal, UFES, Alegre, ES, Brasil - rcecilio@cca.ufes.br \\ ${ }^{5}$ Eng. Agrícola, Dr., Depto. de Engenharia Rural, UFES, Alegre, ES, Brasil - xavier@cca.ufes.br \\ Recebido para publicação: 10/10/2008 - Aceito para publicação: 10/06/2009
}

\begin{abstract}
Resumo
Com o objetivo de determinar a aptidão agroclimática das espécies Pinus elliottii Engelm var. elliottii, Pinus caribaea var. hondurensis Morelet, Pinus taeda Linnaeus e Pinus oocarpa Schiede ex Schltdl no Espírito Santo (ES), foi realizado o zoneamento agroclimático do estado. Utilizaram-se dados meteorológicos do período de 1977 a 2006, provenientes de 96 postos pluviométricos da Agência Nacional de Águas (ANA), sendo 80 localizados dentro do estado e 16 em estados circunvizinhos, de 11 estações meteorológicas pertencentes ao INCAPER (Instituto Capixaba de Pesquisa e Extensão Rural) e de três do INMET (Instituto Nacional de Meteorologia). Os elementos climáticos para determinar a aptidão foram a temperatura do ar e a deficiência hídrica anual para a capacidade de água disponível de $300 \mathrm{~mm}$. De acordo com os resultados, o estado apresenta áreas com aptidão climática para todas as espécies de Pinus, sendo que a espécie Pinus caribaea var. hondurensis apresenta a maior área de aptidão quando comparada com as demais, devido às suas exigências climáticas serem mais restritas.
\end{abstract}

Palavras-chave: Pinus; balanço hídrico; sistema de informação geográfica; zoneamento agroclimático.

\begin{abstract}
Agroclimatic zoning for cultivation of Pinus in the State of Espirito Santo. A agroclimatological zoning was elaborated to determine the suitable areas for growing Pinus elliottii Engelm var. elliottii, Pinus caribaea var. hondurensis Morelet, Pinus taeda Linnaeus and Pinus oocarpa Schiede ex Schltdl in the state of Espírito Santo, Brazil. It was used meteorological data from 1977 to 2006, from eighty rainfall posts of the Water National Agency (ANA) located within the state and sixteen posts surrounding states, eleven weather stations belonging of the Instituto Capixaba de Pesquisa, Assistência Técnica e Extensão Rural (INCAPER) and three weather stations of the Instituto Nacional de Meteorologia (INMET). The climate elements used were air temperature and annual water deficiency, calculated for a soil water storage capacity of $300 \mathrm{~mm}$. The Espírito Santo State showed areas climatically suitable for all species of Pinus, and the species Pinus caribaea var. hondurensis showed the biggest climatically suitable areas compared with the species Pinus elliottii var. elliottii, Pinus oocarpa, Pinus taeda, which due to its climatic requirements, showed lower climatically suitable area.

Keywords: Water balance; climatic zoning, Brazil.
\end{abstract}

\section{INTRODUÇÃO}

De acordo com o levantamento coordenado por Bull et al. (1998), os plantios industriais de Pinus no mundo totalizam 18.319.000 ha, sendo que as maiores áreas cultivadas localizam-se na China, Brasil, Chile e Nova Zelândia.

No Brasil, a implantação de sistemas silviculturais com espécies exóticas tem-se mostrado como uma alternativa econômica sustentável, diminuindo a pressão sobre os ecossistemas naturais, já que permite ofertas de produtos madeiráveis e não-madeiráveis, como a extração de resinas, por exemplo 
(REMADE, 2002). As espécies do gênero Pinus vêm sendo plantadas em escala comercial no Brasil há mais de 30 anos, tornando-se economicamente viável devido a sua grande versatilidade em crescer e produzir madeira nos mais variados tipos de ambiente, bem como a multiplicidade de usos de sua madeira, possibilitando a geração desse recurso natural em todo o território nacional, em substituição às madeiras de espécies nativas (EMBRAPA, 2006).

No Espírito Santo, as espécies que mais se adaptaram às condições climáticas foram o Pinus elliottii var. elliottii, o Pinus caribaea var. hondurensis, o Pinus taeda e o Pinus oocarpa, porém muitas delas ainda não alcançam sua produtividade potencial em determinados locais, devido à influência de fatores térmicos e hídricos que limitam o seu desenvolvimento. Mediante tal fato, é de suma importância a escolha de áreas adequadas para a implantação de cada uma das espécies, de acordo com suas exigências climáticas.

Com o desenvolvimento das indústrias nos setores de papel e celulose, serrados e painéis de madeira, houve um grande consumo de madeira que superou a capacidade de produção, apontando para a necessidade de expandir o reflorestamento para suprir a demanda existente. Porém, a implantação aleatória de povoamentos florestais e a falta de informações sobre o cultivo de Pinus no estado tornam os custos para implantação onerosos, muitas vezes não se conseguindo obter a produtividade esperada.

Comprovada a carência dessas informações, existe uma grande necessidade de estudos mais detalhados a esse respeito, baseados nas condições dos locais de origem do gênero e dados de produtividade em sítios com plantios comerciais, justificando a realização de um zoneamento agroclimático para delimitar áreas com aptidão climática no Espírito Santo.

O zoneamento agroclimático constitui uma importante ferramenta para o planejamento da agricultura com vistas à sua utilização racional, de modo que venha a refletir na otimização dos investimentos. Para isso, contudo, sua atualização se faz necessária (CAMARGO et al., 1974; ROCHA, 1997; SEDIYAMA et al., 2001). Os mapas gerados definem as áreas com potenciais econômicos para a introdução das espécies em uma determinada região geográfica, servindo como base para pequenos, médios e grandes produtores que queiram investir na cultura do Pinus, podendo aumentar assim a eficiência e os lucros dessa atividade.

Diante do exposto, o presente trabalho teve como objetivo definir, por meio de um zoneamento agroclimático, áreas potenciais para implantação de Pinus elliottii var. elliottii, Pinus caribaea var. hondurensis, Pinus taeda e Pinus oocarpa no Espírito Santo, por meio de técnicas de geoprocessamento.

\section{MATERIAL E MÉTODOS}

\section{Área de estudo}

A área contemplada no presente estudo é o estado do Espírito Santo, com área total de $46.184,1 \mathrm{~km}^{2}$, situando-se geograficamente entre os meridianos $39^{\circ} 38^{\prime}$ e $41^{\circ} 50^{\prime}$ de longitude oeste e entre os paralelos $17^{\circ} 52^{\prime}$ e $21^{\circ} 19^{\prime}$ de latitude sul. Apresenta áreas planas e elevadas, sendo que o clima é influenciado de maneira marcante pelo relevo, altitude e exposição das serras. Conforme o sistema de classificação de Köppen, a região enquadra-se nas zonas climáticas A e C, que identificam climas úmidos. No estado são encontrados os subtipos climáticos $\mathrm{Aw}, \mathrm{Am}, \mathrm{Cf}$ e $\mathrm{Cw}$, e também as variações $\mathrm{Cfa}$, Cfb, Cwa e Cwb (SIQUEIRA et al., 2004).

\section{Dados meteorológicos utilizados no estudo}

Foram utilizadas séries históricas de temperaturas médias do ar e total mensal de precipitação pluvial, em escala mensal e anual, considerando-se um período de 30 anos de dados (1977-2006).

Para o Espírito Santo, os dados meteorológicos foram obtidos de 94 pontos de medição, sendo 11 pertencentes à rede de estações meteorológicas do Instituto Capixaba de Pesquisas e Extensão Rural (INCAPER), 3 pertencentes ao Instituto Nacional de Meteorologia (INMET), que medem temperatura do ar e precipitação, e outros 80 pertencentes à Agência Nacional de Águas (ANA), que medem somente a precipitação. Adotaram-se ainda outros 16 postos pluviométricos também pertencentes à ANA, localizados fora do estado, tendo como objetivo minimizar o efeito de borda no processo de interpolação, assim como realizado por Andrade (1998). No total, foram utilizados 110 pontos de medição, cuja distribuição espacial é apresentada na figura 1. 


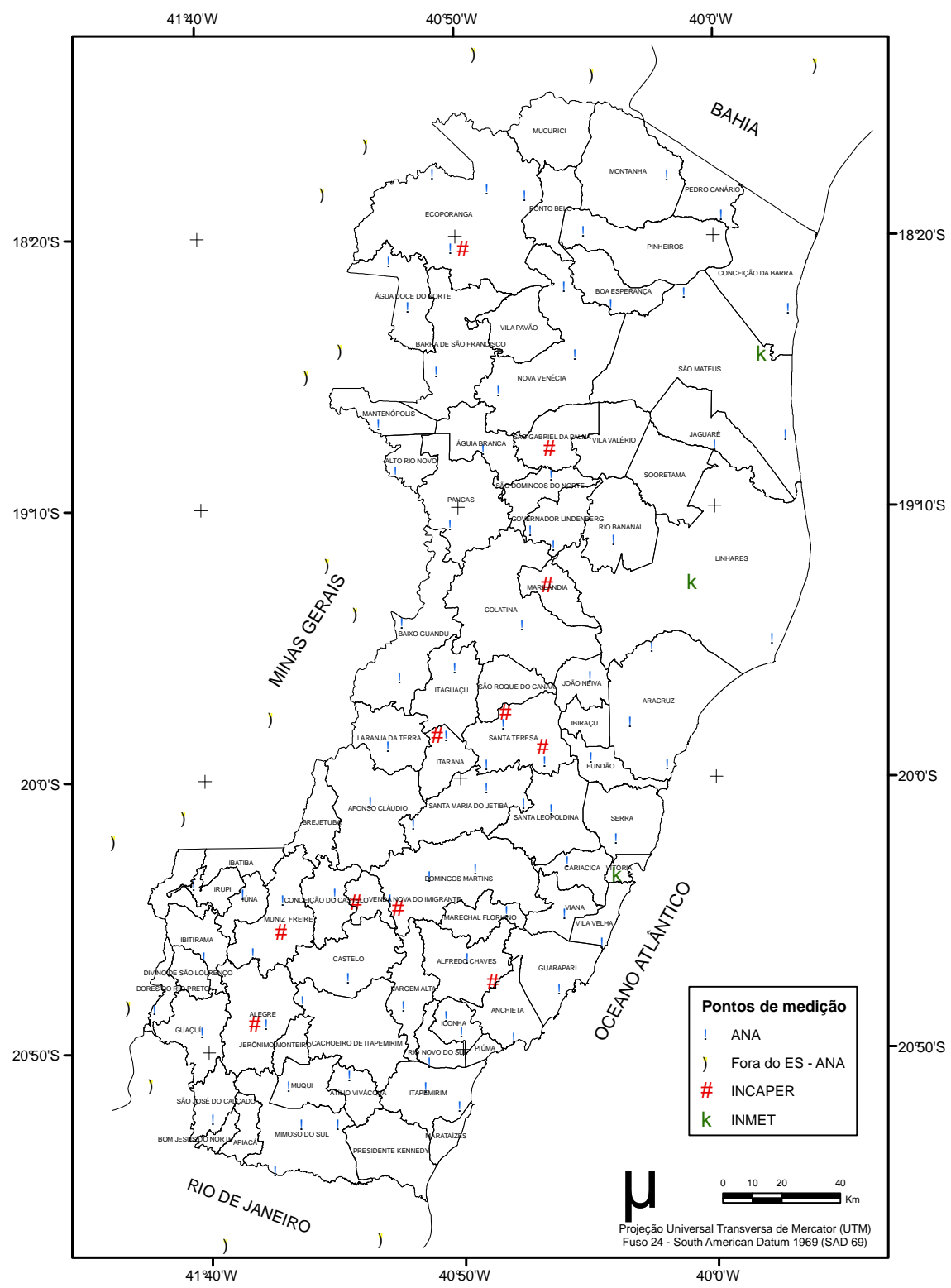

Figura 1. Distribuição espacial dos pontos de medição dos dados climáticos localizadas no Espírito Santo e estados vizinhos.

Figure 1. Spatial distribution of the points of measuring climate data located State of Espirito Santo and neighbor States.

\section{Preenchimento das falhas de precipitação}

Alguns postos de medição da ANA apresentavam falhas de precipitação pluvial nas séries mensais, as quais foram preenchidas utilizando-se o método da ponderação regional, pelo qual são selecionados pelo menos três postos vizinhos daquele que apresentam falhas e que possuem no mínimo dez anos de dados. Adotou-se o método da ponderação regional por ele ser um método simplificado e normalmente o mais utilizado para o preenchimento de séries mensais ou anuais de precipitações pluviométricas (BERTONI; TUCCI, 2002). 
De todos os postos espacializados, foram selecionados três, tomando-se como critério aqueles mais próximos e que estavam numa região climatológica semelhante ao posto a ser estimado, sendo as falhas preenchidas utilizando-se a Equação 1:

$$
\mathrm{Yc}=\frac{1}{3}\left[\frac{\mathrm{X}_{1}}{\mathrm{Xm}_{1}}+\frac{\mathrm{X}_{2}}{\mathrm{Xm}_{2}}+\frac{\mathrm{X}_{3}}{\mathrm{Xm}_{3}}\right] * \mathrm{Ym}
$$

Em que: $\mathrm{Yc}=$ precipitação do posto a ser estimada;

$\mathrm{X}_{1}, \mathrm{X}_{2}$ e $\mathrm{X}_{3}=$ precipitações correspondentes ao mês (ou ano) que se deseja preencher, observadas nos três postos vizinhos;

$\mathrm{Xm}_{1}, \mathrm{Xm}_{2}$ e Xm $\mathrm{X}_{3}=$ precipitação média nas três estações circunvizinhas;

$\mathrm{Ym}=$ precipitação média do posto a ser estimado.

Essa etapa foi fundamental para a homogeneização do período de informações e análise estatística da precipitação na base de dados, garantindo assim uma maior confiabilidade e exatidão das informações na elaboração do trabalho.

\section{Dados de temperatura do ar}

Devido ao reduzido número de dados meteorológicos disponíveis para o Espírito Santo, optou-se por estimar os valores de temperatura do ar a partir de modelos estatísticos desenvolvidos por Castro (2008) ajustados para essa região. As equações foram desenvolvidas através de análises de regressão linear múltipla, adotando-se o modelo linear (Equação 2), que teve como variáveis independentes a altitude, a latitude e a longitude das estações, e como variável dependente a temperatura do ar. Através do modelo, foi possível estimar as temperaturas do ar para todos os postos da ANA, visto que eles fornecem apenas dados pluviométricos.

$$
\gamma_{i}=\beta_{0}+\beta_{1} \text { Alt }+\beta_{2} \text { Lat }+\beta_{3} \text { Long }+\varepsilon_{i}
$$

Em que: $\gamma_{i}=$ temperaturas normais (médias, máximas e mínimas) mensais $(i=1,2, \ldots, 12)$ e anual (i $=13)$, estimadas em ${ }^{\circ} \mathrm{C}$;

Alt $=$ altitude $(\mathrm{m})$;

Lat $=$ latitude, em graus e décimos (entrada com valores negativos);

Long $=$ longitude, em graus e décimos (entrada com valores negativos);

$\beta_{0}, \beta_{1}, \beta_{2}$ e $\beta_{3}=$ parâmetros de regressão;

$\varepsilon_{\mathrm{i}}=$ erro aleatório.

Para determinar os coeficientes das equações, utilizaram-se como base os dados mensais médios de temperaturas máxima, média e mínima do ar, coletados em abrigos termométricos, padrões observados em 14 estações meteorológicas pertencentes ao INCAPER e ao INMET. Consideraram-se para o presente estudo as séries históricas de mesmo tamanho, sem falhas, compreendidas no período de 1977 a 2006 (30 anos).

Para os postos pluviométricos que estavam localizados fora do estado, estimou-se a temperatura média do ar em escala mensal e anual, através de modelos matemáticos, utilizando-se os trabalhos de Assad et al. (2001), Sediyama et al. (2001) e Afonsi et al. (2007), para Bahia, Minas Gerais e Rio de Janeiro, respectivamente.

A temperatura do ar foi espacializada, aplicando-se as equações de regressão à imagem digital de latitude, longitude e altitude do Espírito Santo, em um sistema de informações geográficas. Para os dados de altitude, utilizou-se um modelo digital de elevação, obtido através de dados de radar Shuttle Radar Topography Mission (SRTM), originário da missão de mapeamento do relevo terrestre SRTM, desenvolvido pela National Aeronautics and Space Administration (NASA) e pela National GeospatialIntelligence Agency (NGA) dos Estados Unidos, no ano de 2000. Os resultados dessa missão internacional permitiram a disponibilização de um modelo digital de elevação (MDE) com resolução de (90 m) para toda a América do Sul (SOUZA FILHO, 2003).

Essas imagens encontram-se disponíveis gratuitamente no endereço eletrônico http://www.relevobr.cnpm.embrapa.br e fazem parte de um programa de reconstituição do relevo do país, 
desenvolvido pela Embrapa Monitoramento por Satélites, como nas cartas topográficas, só que de forma digital e homogênea (MIRANDA, 2007).

\section{Balanço hídrico climatológico}

Com os dados de temperatura média do ar e precipitação pluviométrica para todas as localidades, foi calculado o balanço hídrico climático mensal, pelo método proposto por Thornthwaite; Mather (1955), utilizando como auxílio o programa "BHnorm", elaborado em planilha EXCEL por Rolim et al. (1998), assumindo-se uma capacidade máxima de armazenamento de água no solo (CAD) de $300 \mathrm{~mm}$, conforme é indicado para espécies florestais (PEREIRA et al., 2002).

A evapotranspiração potencial foi estimada pelo método de Thornthwaite (1948), que é um método empírico baseado apenas na temperatura média do ar e nas coordenadas geográficas do local. Escolheu-se esse método pela disponibilidade dos dados climáticos e por ter sido desenvolvido para regiões de clima úmido, apresentando boas estimativas para as condições do estado (PEREIRA et al., 2002). Esses coeficientes foram calculados com as normais climatológicas e tornam-se constantes independentes do ano da estimativa da ETP.

\section{Aptidão agrícola para as espécies de Pinus}

O zoneamento agroclimático consiste essencialmente na delimitação das áreas com aptidão para o cultivo das culturas estudadas, no qual estão estabelecidas as condições hídrico-termal ideais para o seu desenvolvimento e consequente produtividade.

Para a cultura do gênero Pinus, selecionaram-se as espécies Pinus elliottii var. elliottii, Pinus caribaea var. hondurensis, Pinus oocarpa e Pinus taeda. No que se refere aos índices térmicos e hídricos exigidos pelas espécies para o seu desenvolvimento, eles foram classificados de acordo com as necessidades de cada uma, dividindo-se em duas classes de aptidão climática - apta e inapta -, como seguem:

- apta: condições térmicas e hídricas da área apresentam-se favoráveis para o bom desenvolvimento e produção da espécie em escala comercial.

- inapta: quando as características normais do clima não se apresentam adequadas à exploração econômica da espécie, devido a limitações severas dos fatores hídricos ou térmicos, ou ambos, com marcante repercussão em sua produção, exigindo para que sejam corrigidas práticas agrícolas dispendiosas.

A classe restrita não foi considerada, pois ela acontece quando a área apresenta limitações hídricas e/ou térmicas, necessitando de irrigação para suprir a deficiência hídrica, ou variedades resistentes ou adaptadas nos casos da limitação ser térmica. Tais práticas de manejo acabam se tornando dificultosas e economicamente inviáveis para a cultura do Pinus.

Com base nos conhecimentos obtidos da cultura sobre Pinus através da Embrapa (1986), foram estabelecidos os parâmetros térmicos das espécies (Tabela 1).

Tabela 1. Faixa de aptidão térmica para as espécies de Pinus.

Table 1. Range of thermal aptitude for the species of Pinus.

\begin{tabular}{lcc}
\hline \multirow{2}{*}{ Cultura } & Regiôes aptas & Regiões inaptas \\
\cline { 2 - 3 } & \multicolumn{1}{c}{ Temperatura média anual (Ta) } \\
\hline Pinus elliottii var. elliottii & $15 \leq \mathrm{Ta} \leq 24^{\circ} \mathrm{C}$ & $15>\mathrm{Ta}>24^{\circ} \mathrm{C}$ \\
Pinus caribaea var. hondurensis & $21 \leq \mathrm{Ta} \leq 27^{\circ} \mathrm{C}$ & $21>\mathrm{Ta}>27^{\circ} \mathrm{C}$ \\
Pinus oocarpa & $13 \leq \mathrm{Ta} \leq 21^{\circ} \mathrm{C}$ & $13>\mathrm{Ta}>21^{\circ} \mathrm{C}$ \\
Pinus taeda & $13 \leq \mathrm{Ta} \leq 19^{\circ} \mathrm{C}$ & $13>\mathrm{Ta}>19^{\circ} \mathrm{C}$ \\
\hline
\end{tabular}

Para definir a aptidão hídrica do Pinus, utilizou-se a deficiência hídrica anual, resultante do balanço hídrico médio realizado por Golfari et al. (1978), que definiram os parâmetros de aptidão por deficiências hídricas anuais para algumas espécies de Pinus (Tabela 2). 
Procedimentos para geração dos mapas de zoneamento agroclimático para o Pinus spp.

$\mathrm{O}$ trabalho foi baseado na sobreposição de mapas que caracterizam a aptidão térmica e a aptidão hídrica favoráveis ao desenvolvimento da cultura do Pinus para o Espírito Santo. Uma vez selecionadas as espécies e definidos seus índices climáticos, utilizou-se o software ArcGis 9.2/ArcMap para realizar os cruzamentos e gerar todos os mapas digitais. O esquema de todas as operações envolvidas no processo de manipulação e execução das diversas etapas que culminaram nos mapas finais do zoneamento agroclimático para as espécies de Pinus é apresentado na figura 2.

Tabela 2. Faixa de aptidão hídrica para as espécies de Pinus.

Table 2. Range of water aptitude for the species of Pinus.

\begin{tabular}{lcc}
\hline \multirow{2}{*}{ Cultura } & \multicolumn{1}{c}{ Regiões aptas } & Regiões inaptas \\
\cline { 2 - 3 } & \multicolumn{1}{c}{ Defiência hídrica anual } \\
\hline Pinus elliottii var. elliottii & $\leq 50 \mathrm{~mm}$ & $>50 \mathrm{~mm}$ \\
Pinus caribaea var. hondurensis & $\leq 200 \mathrm{~mm}$ & $>200 \mathrm{~mm}$ \\
Pinus oocarpa & $\leq 200 \mathrm{~mm}$ & $>200 \mathrm{~mm}$ \\
Pinus taeda & $\leq 50 \mathrm{~mm}$ & $>50 \mathrm{~mm}$ \\
\hline
\end{tabular}

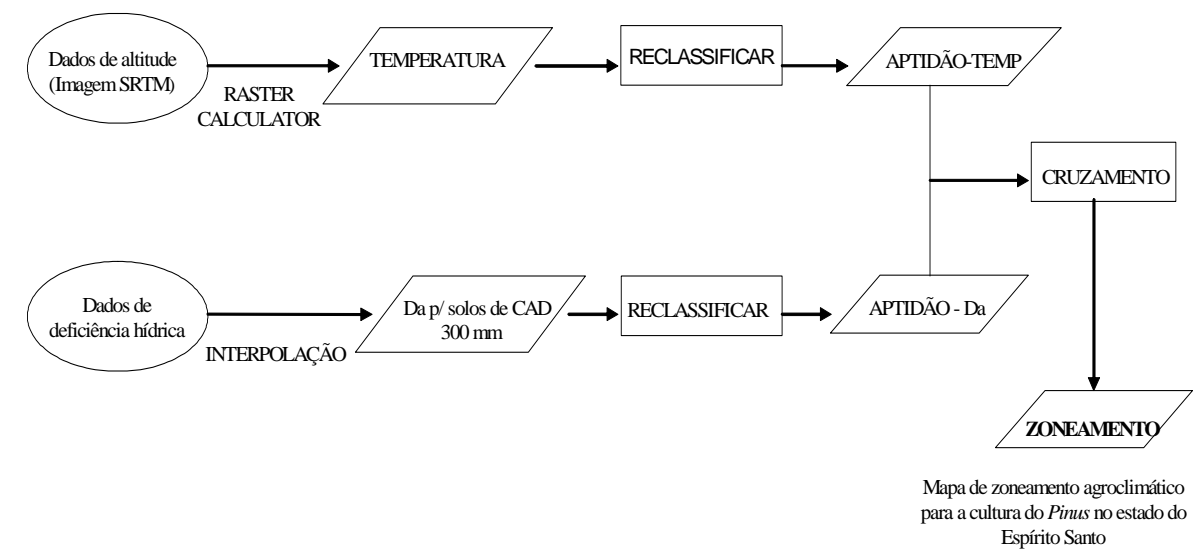

Figura 2. Fluxograma das etapas necessárias para a obtenção dos mapas de zoneamento agroclimático da cultura do Pinus no Espírito Santo, em que TEMP: temperatura média anual, Da: deficiência hídrica anual e CAD: capacidade de armazenamento disponível.

Figure 2. Flowchart of steps necessary to obtain the maps of agroclimatic zoning of the culture of Pinus in the state of Espirito Santo, where TEMP: average annual temperature, Da: water annual deficiency and CAD: storage available capacity.

Primeiramente, realizou-se o mosaico das imagens SRTM, gerando uma imagem "raster" da altitude para o Espírito Santo, e a partir das coordenadas geográficas do limite do estado realizou-se uma interpolação linear através do módulo "ArcToolbox - Spatial Analyst Tools - Interpolation", obtendo assim as imagens "raster" de latitude e longitude.

Diante das imagens digitais de altitude, latitude e longitude, aplicaram-se os coeficientes obtidos através das equações de regressão ajustadas ao modelo linear (Equação 1). Através do módulo "Spatial Analyst - Raster Calculator", obteve-se o mapa digital da espacialização da temperatura média anual do ar.

De posse do mapa de temperatura média anual, procedeu-se, através do módulo "ArcToolbox Spatial Analyst Tools - Reclass”, a sua reclassificação, considerando as faixas de aptidão conforme os índices térmicos estabelecidos para cada espécie.

De acordo com os dados de deficiência hídrica do solo, obtidos através do balanço hídrico climatológico (THORNTHWAITE; MATHER, 1955), realizou-se a espacialização desses valores, estipulando as áreas com deficiência hídrica para todo o estado. Para isso, procedeu-se a interpolação dos dados, através do método da krigagem modelo esférico, utilizando-se rotinas específicas de um SIG 
implementado através do software ArcGis 9.2/ArcMap pelo módulo "ArcToolbox - Spatial Analyst Tools - Interpolation", o qual deu origem ao mapa temático que representa as condições de deficiência hídrica do estado.

Uma vez estabelecido o mapa de classes de deficiência hídrica anual para o Espírito Santo, foram gerados os mapas de zonas de deficiência hídrica anual para cada espécie, de acordo com as suas faixas de aptidão hídrica, por intermédio da reclassificação através do módulo "Spatial Analyst Tools Reclass".

Finalmente, os mapas de zoneamento agroclimático para o Pinus spp. foram obtidos através do cruzamento dos mapas de temperatura média anual e deficiência hídrica anual já reclassificados, conforme fluxograma da figura 2 .

\section{RESULTADOS E DISCUSSÃO}

\section{Mapa de temperatura média anual do ar}

Trabalhos realizados por Sediyama et al. (2001), Pezzopane et al. (2004), Valadares et al. (2005) e Medeiros et al. (2005) ressaltam a importância da inclusão do fator relevo na espacialização da temperatura do ar, contribuindo muito para melhorar os resultados obtidos em relação à simples interpolação de dados medidos em estações meteorológicas.

A figura 3 mostra a espacialização da temperatura média anual do ar para todo o estado, utilizando um MDE gerado a partir de dados de radar SRTM e os coeficientes da equação de estimativa (CASTRO, 2008), sendo possível detectar com bastante sensibilidade a variação do relevo.

Ainda de acordo com a figura 3 , a parte do estado com altitude entre 0 e $300 \mathrm{~m}$, compreendendo quase todo o norte e o vale do Rio Itapemirim ao sul, além de toda a faixa litorânea, apresenta temperatura média anual acima de $24^{\circ} \mathrm{C}$, enquanto a região serrana ao sul apresenta, em sua maior parte, temperatura média anual entre 18 e $22^{\circ} \mathrm{C}$.

\section{Mapa de deficiência hídrica anual acumulada}

O mapa de espacialização da deficiência hídrica anual é de extrema importância em trabalhos de zoneamentos agrícolas, indicando o grau de deficiência de água no solo.

A figura 4, de uma maneira geral, aponta que a região norte possui uma área maior de deficiência hídrica quando comparada com a sul e a região serrana, onde os locais mais elevados apresentam deficiência hídrica anual abaixo de $50 \mathrm{~mm}$. No extremo norte, a deficiência hídrica anual é superior a 250 $\mathrm{mm}$, e na região oeste, no Vale do Rio Doce, foram encontrados os maiores valores de deficiência, com média próxima a $400 \mathrm{~mm}$, resultante da baixa disponibilidade hídrica e da alta demanda evaporativa.

\section{Mapas de zoneamento agroclimático para algumas espécies de Pinus}

Foram identificadas as áreas com aptidão para implantação de povoamentos florestais de produção e reflorestamento em todo o estado do Espírito Santo, de acordo com as bases estabelecidas para esse zoneamento.

O Pinus elliottii var. elliottii é a espécie mais indicada para implantação nas regiões com altitudes acima de 500 metros, onde as temperaturas são mais amenas. As áreas com aptidão para o plantio dessa espécie abrangem grande parte do sul do estado, principalmente aquelas áreas mais elevadas, onde a temperatura do ar é mais baixa e a deficiência hídrica pequena (Figura 5).

A figura 6, apresenta o zoneamento agroclimático para a espécie Pinus oocarpa, que se desenvolve bem em regiões que apresentam altitudes superiores a 500 metros, consequentemente as mais frias. As áreas climaticamente aptas para a implantação dessa espécie são maiores quando comparadas ao Pinus taeda (Figura 7), sendo que as duas espécies apresentam exigências térmicas muito próximas. Com relação à deficiência hídrica, o Pinus oocarpa consegue tolerar deficiência hídrica até $200 \mathrm{~mm}$, enquanto o Pinus taeda tolera somente até $50 \mathrm{~mm}$.

Ainda de acordo com a figura 7, observa-se que a espécie Pinus taeda pode se desenvolver apenas em uma pequena porção da região serrana do Espírito Santo, abrangendo os municípios de Venda Nova do Imigrante, Domingos Martins, Santa Maria de Jetibá, Conceição do Castelo e parte do Caparaó. Isso porque essas localidades possuem altitudes mais elevadas com temperaturas mais amenas, 
apresentando, portanto deficiência hídrica e evapotranspiração real menor quando comparadas com as localidades de altitudes menores.

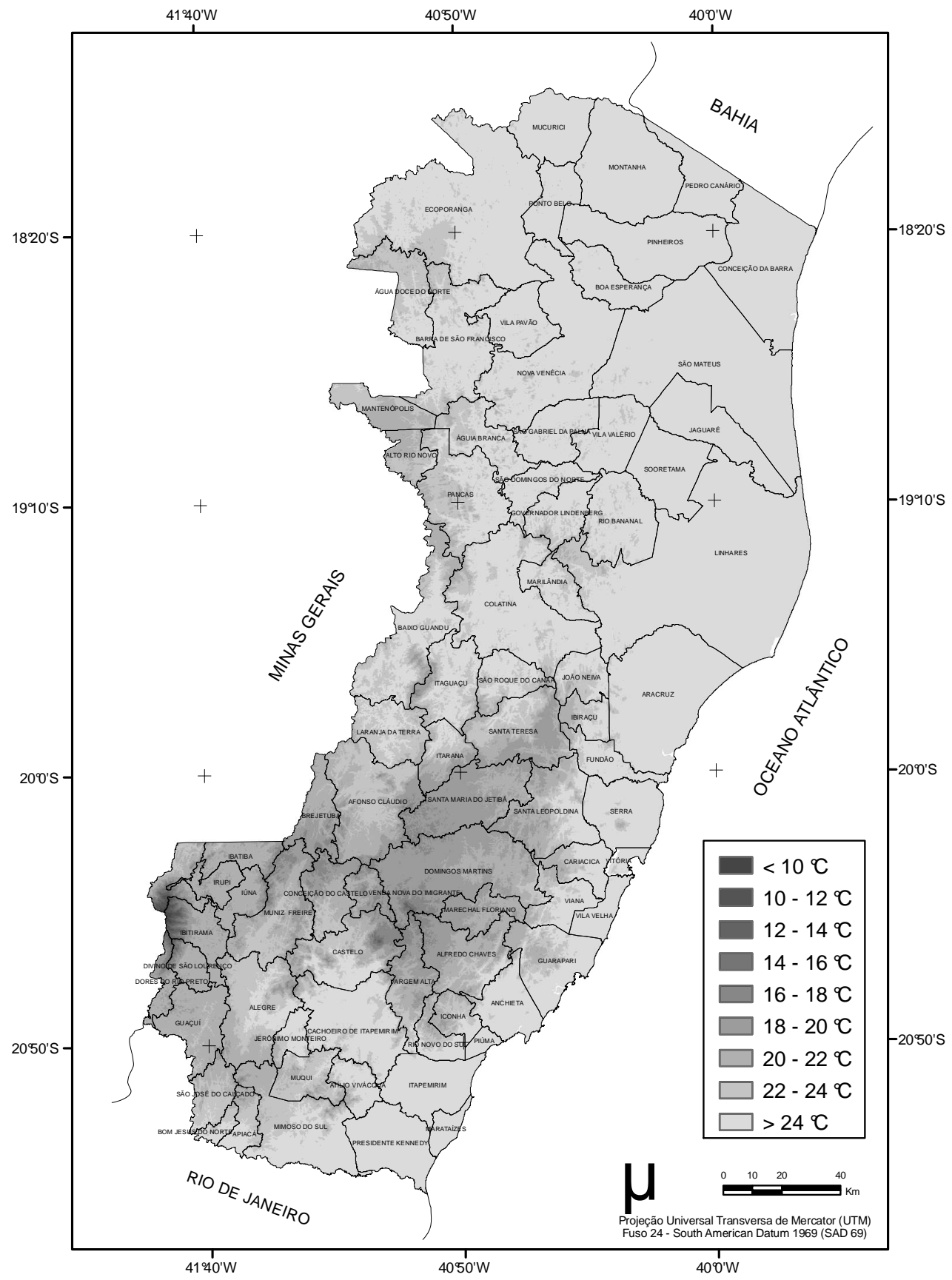

Figura 3. Espacialização das estimativas dos valores da temperatura média anual do ar para o Espírito Santo, utilizando-se equações múltiplas lineares e modelo digital de elevação através do SRTM.

Figure 3. Spacialization of estimates of the average air annual temperature for the state of Espírito Santo, using linear multiple equations and digital elevation model by SRTM. 
Como a espécie de Pinus taeda não tolera deficiência hídrica maior que $50 \mathrm{~mm}$, ela não é indicada para implantação na maior parte do estado, pois este apresenta deficiência hídrica elevada em quase todo o seu território.

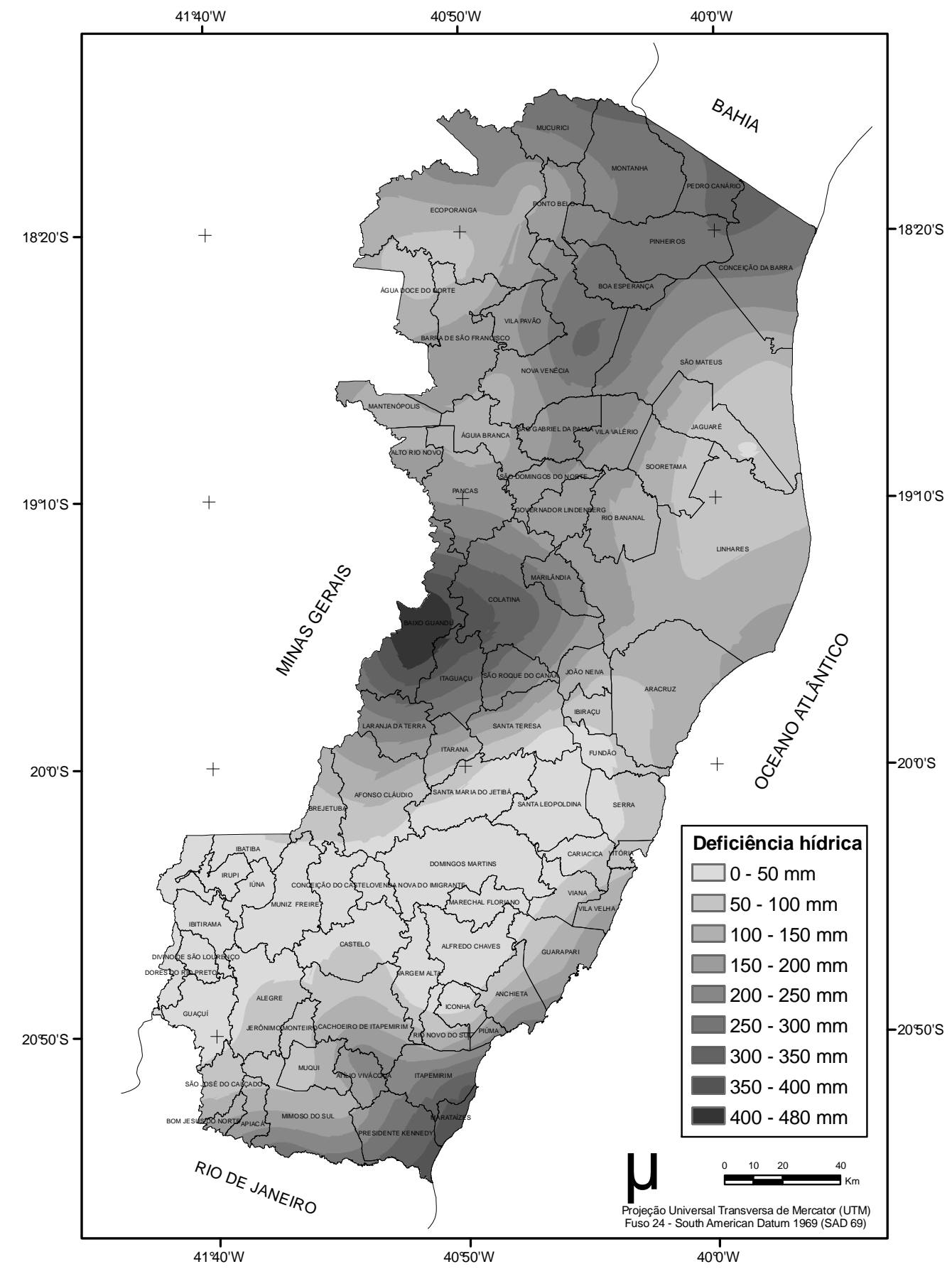

Figura 4. Mapa da deficiência hídrica anual acumulada para o Espírito Santo.

Figure 4. Map of annual water deficit accumulated for the State of Espirito Santo.

O Pinus caribaea var. hondurensis (Figura 8) possui características de clima quente, podendo ser introduzido praticamente ao longo de todo o litoral e na maioria dos municípios do norte do Espírito 
Santo, o que não ocorre para o Pinus elliottii var. elliottii, Pinus oocarpa e Pinus taeda. REMADE (2002) confirma tal fato, recomendando a implantação do Pinus caribaea var. hondurensis em quase todo o território do Brasil, incluindo toda a faixa litorânea.

Com base nas exigências climáticas dessa espécie, ela pode ser implantada em regiões quentes, porém com deficiência hídrica menor que $200 \mathrm{~mm}$.

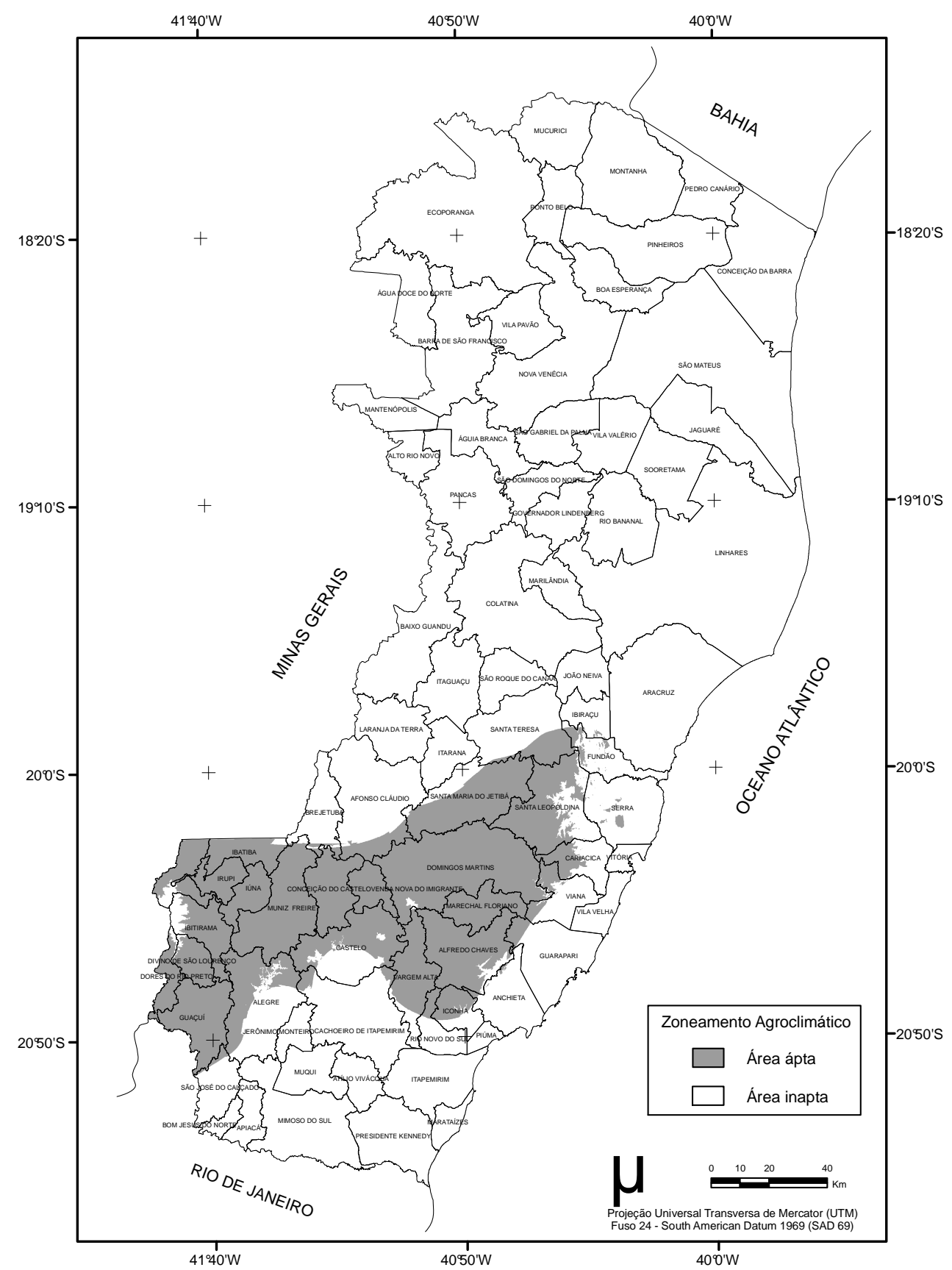

Figura 5. Zoneamento agroclimático para a espécie de Pinus elliottii var. elliottii no estado do Espírito Santo.

Figure 5. Agroclimatic zoning for the specie of Pinus elliottii var. elliottii in the State of Espírito Santo. 


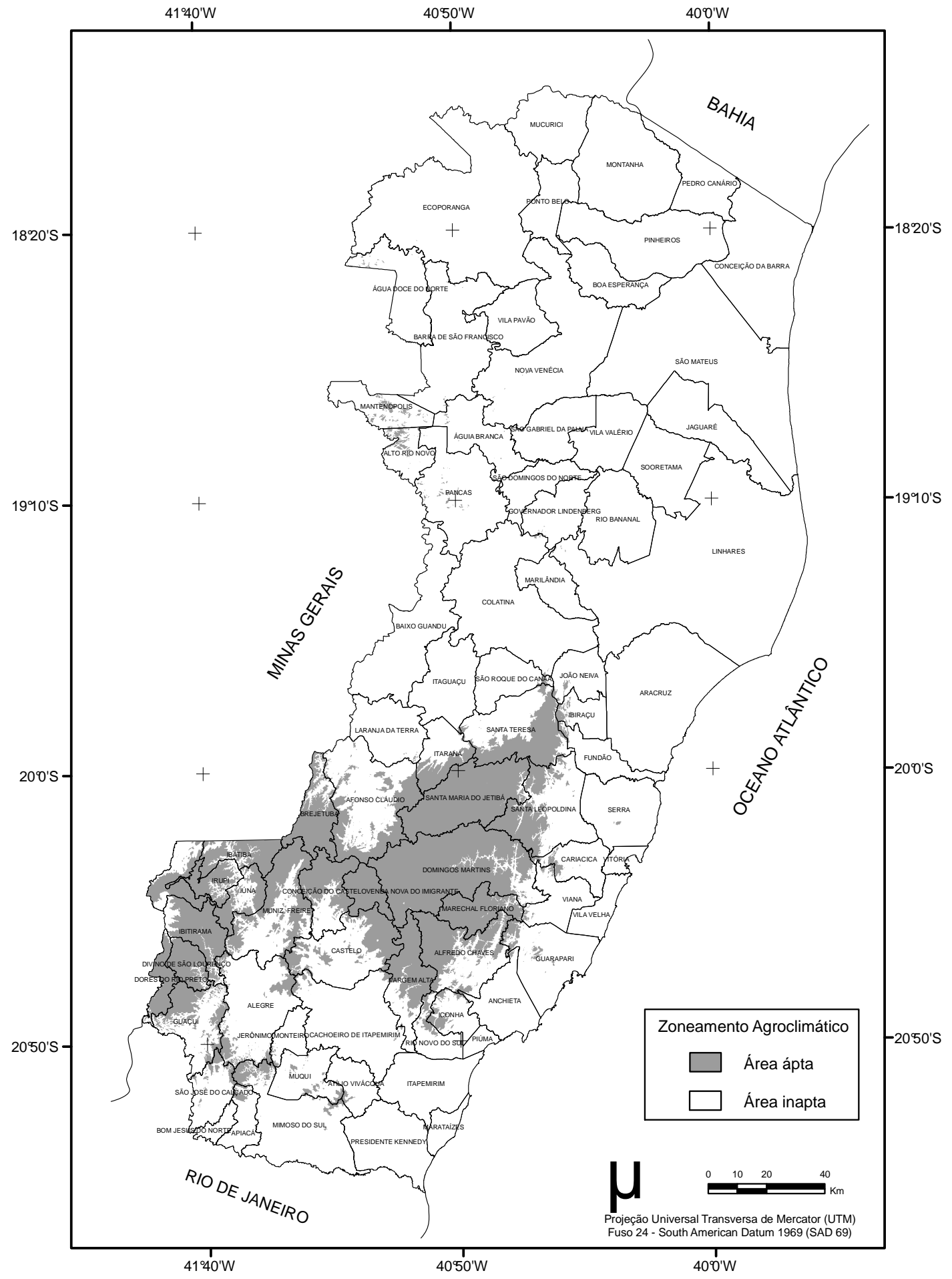

Figura 6. Zoneamento agroclimático para a espécie de Pinus oocarpa no estado do Espírito Santo. Figure 6. Agroclimatic zoning for the specie of Pinus oocarpa in the State of Espírito Santo. 


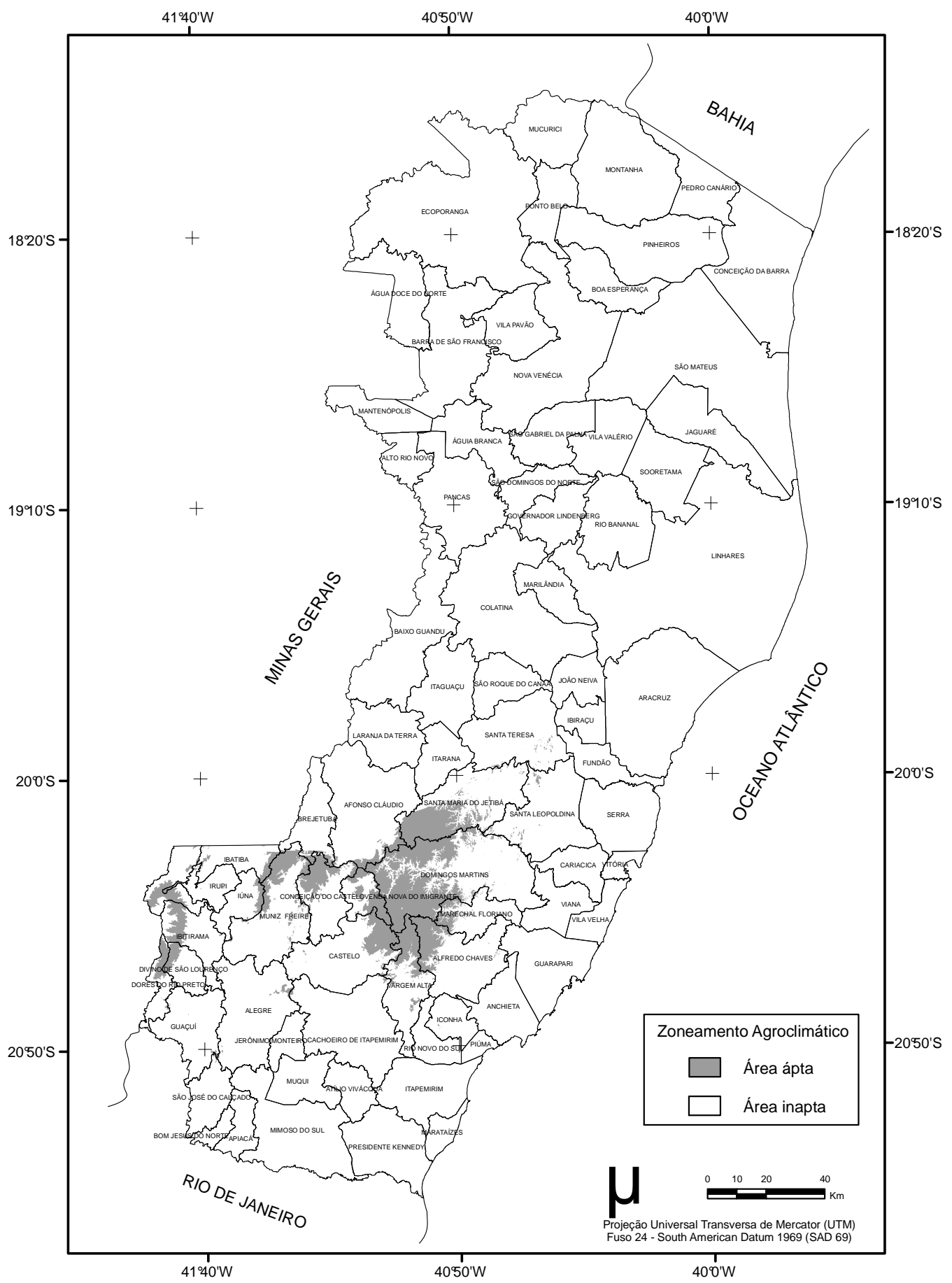

Figura 7. Zoneamento agroclimático para a espécie de Pinus taeda no estado do Espírito Santo. Figure 7. Agroclimatic zoning for the specie of Pinus taeda in the State of Espírito Santo.

Nas áreas ao norte do estado e naquelas mais baixas próximas do litoral, é possível observar, através do zoneamento agroclimatológico, que a deficiência hídrica e a temperatura apresentam valores elevados, influenciando o desenvolvimento da cultura, sendo então consideradas inaptas para o plantio das espécies Pinus elliottii var. elliottii, Pinus oocarpa e Pinus taeda. 


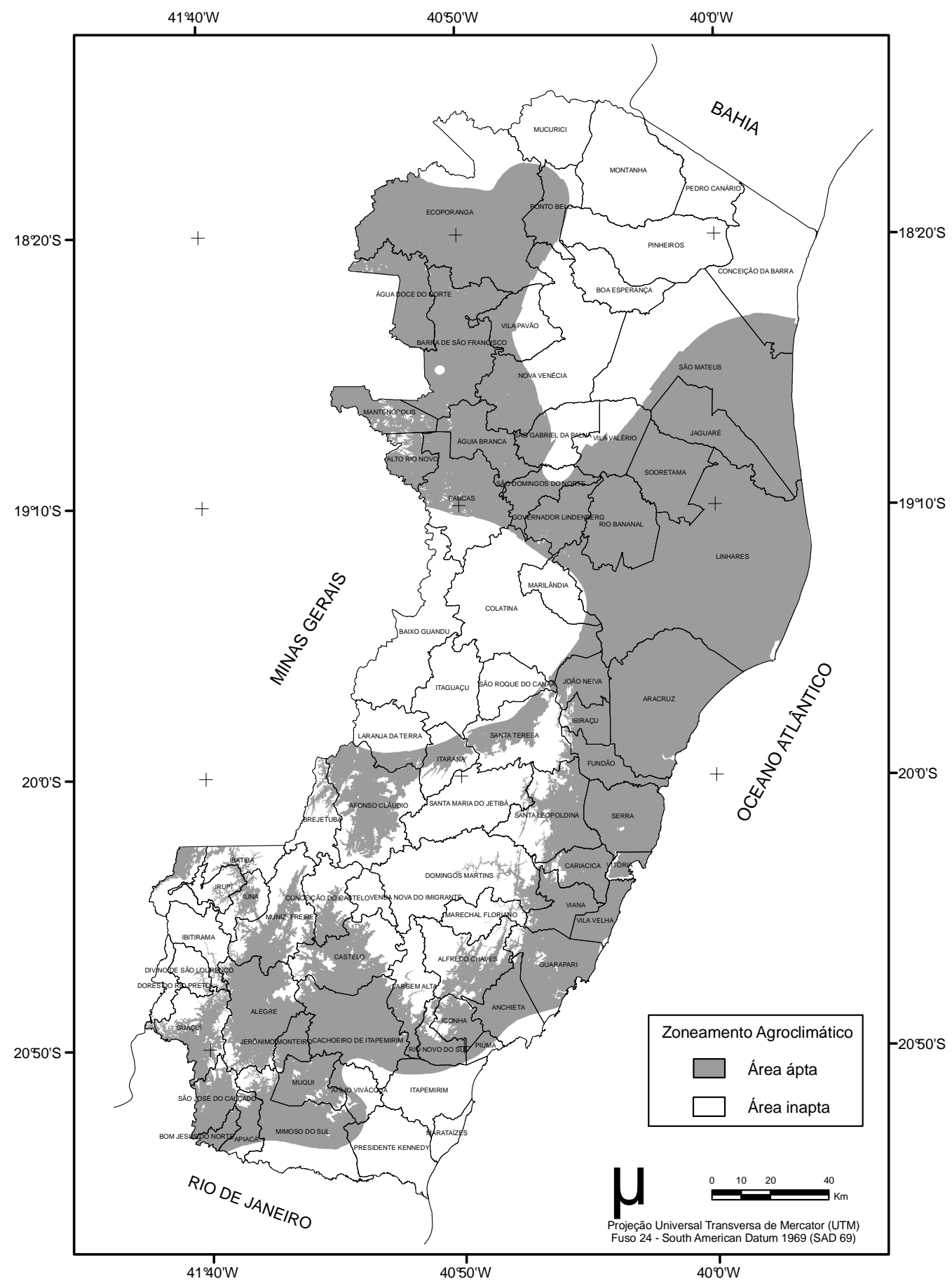

Figura 8. Zoneamento agroclimático para a espécie de Pinus caribaea var. hondurensis no estado do Espírito Santo.

Figure 8. Agroclimatic zoning for the specie of Pinus caribaea var. hondurensis in the State of Espírito Santo.

Na tabela 3 é apresentada a porcentagem de áreas aptas e inaptas para a implantação do Pinus spp. no Espírito Santo. 
Tabela 3. Áreas (\%) de aptidão para a implantação de Pinus spp. no estado do Espírito Santo. Table 3. Áreas (\%) of aptitude for implantation of Pinus spp. in the State of Espirito Santo.

\begin{tabular}{lcc}
\hline Espécies & Áreas aptas (\% do total) & Áreas inaptas (\% do total) \\
\hline Pinus elliottii var. elliottii & 18,2 & 81,8 \\
Pinus caribaea var. hondurensis & 54,4 & 45,6 \\
Pinus oocarpa & 15,6 & 84,4 \\
Pinus taeda & 4,2 & 95,8 \\
\hline
\end{tabular}

O resultado da tabela 3 mostra que o Pinus caribaea var. hondurensis pode ser implantado em $54,4 \%$ da área total do estado. Esse valor elevado foi devido à baixa restrição em relação às exigências climáticas, quando comparado com as outras espécies. Todavia, o Pinus taeda é o que apresenta a menor porção de área apta para a sua implantação, devido às suas restrições hídricas e térmicas.

\section{CONCLUSÕES}

- O Espírito Santo apresenta áreas com aptidão climática para todas as espécies de Pinus, de acordo com as bases estabelecidas para este zoneamento.

- Devido à espécie de Pinus taeda necessitar de temperaturas mais baixas e não tolerar deficiência hídrica, a área considerada apta para sua implantação é muito pequena, limitando-se às regiões mais elevadas do estado.

- Para as espécies Pinus elliottii var. elliottii e Pinus oocarpa, as áreas propícias para a sua implantação são quase similares, porém a área com aptidão para o Pinus elliottii var. elliottii é mais abrangente, devido a essa espécie se desenvolver em locais com temperaturas mais elevadas.

- Pelo fato das espécies de Pinus elliottii var. elliottii, Pinus oocarpa e Pinus taeda apresentarem limitações climáticas bastante restritas e incompatíveis com a maioria dos índices climáticos do estado, as áreas consideradas impróprias para a sua implantação são superiores às áreas consideradas aptas.

- Para a espécie Pinus caribaea var. hondurensis, as áreas com aptidão para implantação no estado são mais abrangentes que as inaptas. As áreas aptas compreendem principalmente grande parte do norte $\mathrm{e}$ locais próximos ao litoral, onde a deficiência hídrica e as temperaturas do ar são bem mais elevadas.

\section{REFERÊNCIAS}

AFONSI, R. R.; PINTO, H. S.; ZULLO JUNIOR, J.; CORAL, G.; ASSAD, E. D.; EVANGELISTA, B. A.; LOPES, T. S. de S.; MARRA, E.; BEZERRA, H. S.; HISSA, H. R.; FIGUEIREDO, A. F. de; SILVA, G. G. da; SUCHAROV, E. C.; ALVES, J.; MARTORANO, L. C.; ANDRÉ, R. G. B.; BASTOS, W. E. de. Zoneamento climático da cultura do café (Coffea arabica) no estado do Rio de Janeiro. Disponível em: 〈http://www.cpa.unicamp.br/cafe/index.shtml-relat_rj.html>. Acesso em: 25/06/2007.

ANDRADE, L. A. Classificação ecológica do território brasileiro situado a leste do meridiano de $\mathbf{4 4}^{\circ}$ oeste e ao norte do paralelo de $\mathbf{1 6}^{\circ}$ sul: uma abordagem climática. $147 \mathrm{f}$. Tese. (Doutorado em Ciência Florestal) - Universidade Federal de Viçosa, Viçosa, MG, 1998.

ASSAD, E. D.; EVANGELISTA, B. A.; SILVA, F. A. M. da.; CUNHA, S. A. R. da.; ALVES, E. R.; LOPES, T. S. S.; PINTO, H. S.; ZULLO JUNIOR, J. Zoneamento agroclimático para a cultura de café (Coffea arabica L.) no estado de Goiás e sudoeste do estado da Bahia. Revista Brasileira de Agrometeorologia, Santa Maria, v. 9, n.3, p. 510-518, 2001.

BERTONI, J.C.; TUCCI. C. E. M. Precipitação In: TUCCI, C. E. M. (Org.). Hidrologia: ciência e aplicação. 3. ed. Porto Alegre: UFRGS/ABRH, 2002. p. 177-231.

BULL, G.; MABEE, W.; SCHARPENBERG, R. Global fibre supply model. Rome: Food and Agriculture Organization of the United Nations, 1998. 72 p.

CAMARGO, Â. P.; PINTO, H. S.; BRUNINI, O.; PEDRO JR, M. J.; ORTOLANI, A. A.; ALFONSI, R. R. Aptidão climática de culturas agrícolas. In: São Paulo: Secretaria da Agricultura. Zoneamento agrícola do Estado de São Paulo. São Paulo: CATI, 1974. v. 1, p 109-49. 
CASTRO, F. S. Zoneamento agroclimático para a cultura do Pinus no Estado do Espírito Santo. 121f. Dissertação (Mestrado em Produção Vegetal) - Universidade Federal do Espírito Santo, Alegre, ES, 2008.

EMPRESA BRASILEIRA DE PESQUISA AGROPECUÁRIA (EMBRAPA). Centro Nacional de Pesquisa de Florestas. Zoneamento ecológico para plantios florestais no estado do Paraná. Brasília, DF: EMBRAPA - DDT, 1986. 89 p. (Documentos, n. 17).

\footnotetext{
Sistema de produção. Disponível em:

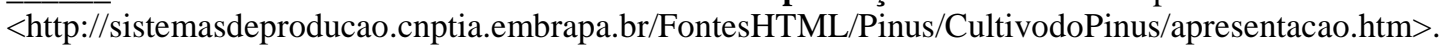
Acesso em: 25/11/2006.
}

GOLFARI, L.; CASER, R. L.; MOURA, V. P. G. Zoneamento ecológico esquemático para reflorestamento no Brasil; ( $2^{\mathrm{a}}$ aproximação). Belo Horizonte: Centro de Pesquisa Florestal da Região do Cerrado, 1978. 66p. (PRODEPEF, Série Técnica, n. 11).

MEDEIROS, S. de. S.; CECÍlIO, R. A.; MELO JUNIOR, J. C. F. de; SILVA JUNIOR, J. L. C. da. Estimativa e espacialização das temperaturas do ar mínimas, médias e máximas na Região Nordeste do Brasil. Revista Brasileira de Engenharia Agrícola e Ambiental, Campina Grande, v. 9, n. 2, p. 247$255,2005$.

MIRANDA, E. E. de. (Coord.). Brasil em relevo. Campinas: EMBRAPA Monitoramento por Satélite, 2005. Disponível em: 〈http://www.relevobr.cnpm.embrapa.br〉. Acesso em: 16/03/2007.

PEREIRA, A. R.; ANGElOCCI, L. R.; SENTElHAS, P. C. Agrometeorologia: fundamentos e aplicações práticas. Guaíba: Agropecuária, 2002. 478 p.

PEZZOPANE, J. E. M.; SANTOS, E. A.; ELEUTÉRIO, M. M.; REIS, E. F. dos; SANTOS, A. R. dos. Espacialização da temperatura do ar no estado do Espírito Santo. Revista de agrometeorologia, Santa Maria, n. 1, v. 12 p. 151-158, 2004.

REVISTA DA MADEIRA (REMADE). n. 12, dez. 2002. Disponível em: <http://www.remade.com.br/pt/revista_capa.php?edicao=68>. Acesso em: 26/01/2007.

ROCHA, J. S. M. da. Manual de projetos ambientais. Brasília, DF: MMA, 1997. 446 p.

ROLIM, G. S.; SENTELHAS, P. C.; BARBIERI, V. Planilhas no ambiente EXCEL para os cálculos de balanços hídricos: normal, sequencial, de cultura e de produtividade real e potencial. Revista Brasileira de Agrometeorologia, Santa Maria, v. 6, p. 133-137, 1998.

SEDIYAMA, G. C.; MELO JUNIOR, J. C. F. de.; SANTOS, A. R. dos.; RIBEIRO, A.; COSTA, M. H.; HAMAKAWA, P. J.; COSTA, J. M. N. da.; COSTA, L. C. Zoneamento agroclimático do cafeeiro (Coffea arabica L.) para o estado de Minas Gerais. Revista Brasileira de Agrometeorologia, Santa Maria, v. 9, n. 3, p. 501-509, 2001.

SIQUEIRA, J. D. P.; LISBOA, R. S.; FERREIRA, A. M.; SOUZA, M. F. R. de; ARAÚJO, E. de; LISBÃO JÚNIOR, L.; SIQUEIRA, M. de. M. Estudo ambiental para os programas de fomento florestal da Aracruz Celulose S. A. e extensão florestal do governo do estado do Espírito Santo. Floresta, Curitiba, Edição especial, p. 3-67, nov/2004.

SOUZA FILHO, C. R. O relevo das Américas como nunca antes visto. Infogeo, Curitiba, n. 30, p. 54-58, 2003.

THORNTHWAITE, C. W. An approach toward a rational classification of climate. Geographical Review, New York, v. 38, p. 55-94, 1948

THORNTHWAITE, C.W.; MATHER, J. R. The water balance: publications in climatology. New Jersey: Drexel Institute of Technology, 104 p. 1955.

VALLADARES, G. S.; MARIN, F. R.; OSHIRO. O. T.; GOUVÊA, J. R. F. Uso de Imagens de Radar na Estimativa da Temperatura do Ar. In: SIMPÓSIO BRASILEIRO DE SENSORIAMENTO REMOTO, 2005, Goiânia. Anais...Goiânia: INPE, 2005. p. 309-311. 
